\title{
CRS Workshop Report: Using an In Vitro-In Vivo Correlation (IVIVC) to Meet Challenges in Global Delivery
}

\author{
Mario A. Gonzalez ${ }^{1}$ and Vivian A. Gray ${ }^{2}$ \\ 'P'Kinetics International, Inc., Pembroke Pines, FL, USA \\ ${ }^{2}$ V. A. Gray Consulting, Inc., Hockessin, DE, USA
}

$\mathrm{T}$ he Controlled Release Society Educational Workshop on IVIVC was held on Saturday, July 20, 2013, in Honolulu, $\mathrm{HI}$, and was designed to provide a stimulus to pharmaceutical companies to develop in vitro-in vivo correlations (IVIVC). The workshop was sponsored by the SOTAX Group and Simulations Plus, Inc. The Planning Committee was Tapash Ghosh, FDA, Vivian Gray, and Mario Gonzalez.

Vivian A. Gray, editor of Dissolution Technologies and Workshop Chair, opened the workshop by summarizing the objectives of the workshop. She noted that an expanding global market increases the demand for new drugs and controlled-release drug formulations. The cost of developing products for new markets can be greatly reduced if the number of bioavailability and bioequivalence studies can be reduced. Biowaivers obtained through the establishment of valid IVIVCs will be a major contribution to the reduction in drug development costs. She suggested that case studies of biowaivers using IVIVC should be discussed in detail, and encouraged input from the attendees. The importance of developing a biorelevant in vitro test for a drug product was also discussed.

Dr. Mario A. González, President of P'Kinetics International and Adjunct Professor at the University of Florida, addressed the topic Traditional IVIVC and IVIVR Based on Deconvolution. His talk highlighted methods to establish an IVIVC using deconvolution methods such as the Wagner-Nelson equation or numerical deconvolution thus allowing conversion of a plasma concentration profile from a clinical bioavailability study into an absorption profile. The absorption profile can then be compared with the dissolution profile for the same formulation. Ideally, a point-to-point comparison between the in vitro and in vivo data can be conducted and a correlation can be established, resulting in an IVIVC. Such a correlation must be justified using at least three formulations with different dissolution profiles. The validation criteria to establish this correlation or IVIVC is strictly defined in an FDA guidance (1). Three levels of correlation are listed in General Chapter <1088> of the USP (2) as well as in the FDA guidance. The advantages and disadvantages of the three levels of IVIVC were also discussed. If a validated IVIVC cannot be established, it is possible that an in vitro-in vivo relationship (IVIVR) will still be a valuable contribution to formulation scientists developing new extended-release products.
Dr. Sanjay Garg, Professor at the University of South Australia, presented on the Development of a BioRelevant In Vitro Test for the IVIVC. He discussed the importance of dissolution testing in formulation development as well as the critical role it plays in the release of different product batches or lots. Further, dissolution testing is an important regulatory requirement and a major factor in quality control and stability testing. It is the most direct method to ensure lot-to-lot consistency of a manufacturing process as well as the best way to identify critical manufacturing variables. A biorelevant dissolution method has the further advantage of serving as a predictor of bioavailability. Identifying a dissolution method early in the preformulation stage can be a major aid to a formulation group. Dr. Garg also discussed the lack of good dissolution methods for non-oral dosage forms such as buccal/sub-lingual tablets, vaginal delivery systems, and drug-eluting stents. Multipurpose prevention technologies (MPTs) for sexual and reproductive health such as intravaginal rings and diaphragms are especially in need of good dissolution methodology. Identifying the proper dissolution medium for a biorelevant dissolution method can be challenging, but attention should be paid to previous published work to facilitate the development of biorelevant dissolution methods. A final challenge to the audience was to consider whether a dissolution test should be designed to be "highly biosimulating or a test that provides a good IVIVC."

The next speaker was Johannes Krämer, of PHAST GMBH in Germany. His topic was Global Applications of IVIVC in Formulation Development and to Support Biowaivers. He began his presentation by reviewing the basic requirements for dissolution testing from WHO, FDA, and EU. USP Apparatus 4 was described as a tool used for in vitro testing in IVIVC development. He went into detail regarding IVIVC expectations for immediate-release products based on the BCS, of which only BCS Class 2 is a possibility. However, if the dose is very high, it is not likely to provide an IVIVC. He gave examples of how in vitro testing can be matched to in vivo to show rank order-not a substitute for IVIVC but useful to establish a relationship between in vitro and in vivo data. An established rank order can be used to demonstrate that a dissolution medium has been chosen correctly. Examples of Level A correlations were shown, and statistical moment theory/ mean time was discussed as an in vivo parameter that can 
be used to establish a relationship with in vitro dissolution data.

Samir Haddouchi, Managing Director of SPS Pharma Services, France, was the next speaker in the program and addressed the topic IVIVC: But Using What In Vitro

Data? He presented some insights about how in vitro data should be used to establish a relevant IVIVC. Mr. Haddouchi described how in vitro dissolution profiles are often used without a complete understanding of their meaning. From the beginning, it is critical to define the aim of an in vitro dissolution method (e.g., formulation development, IVIVC, or Quality Control). He emphasized that one should keep in mind that the techniques used (paddle, flowthrough cell, etc.) are only "very simple" models trying to mimic a complex physiological process. The presentation highlighted the fact that the in vitro profiles can represent either the dissolution rate of the active ingredient or the release rate from the formulation. A more frequent use of API characterization tools such as intrinsic and apparent dissolution (Eur. Ph. §2.9.29 and 2.9.43, respectively) can be of great help in achieving such a goal. A case study was then shared with the audience showing how the dissolution testing of a simple immediate-release dosage form can be challenging in obtaining an in vitro profile able to predict the in vivo performance of a dosage form.

Grazyna Fraczkiewicz, Team Leader for Simulation Studies, spoke of the software available at Simulations Plus, Inc., that can be used to develop an IVIVC. She discussed the program GastroPlus and its use in a Mechanistic Deconvolution Method to Arrive at an IVIVC. GastroPlus is a simulation software package that provides insight into how various phenomena interact to produce a drug's PK/PD behavior. The software's IVIVC module provides an option to choose between traditional deconvolution methods (Loo-Riegelman, Wagner-Nelson, numerical deconvolution) and mechanistic deconvolution to establish an IVIVC. Some traditional deconvolution methods correlate in vitro dissolution with bioavailability parameters. This is not truly representative of a correlation between in vitro and in vivo release because bioavailability is affected by a combination of factors such as in vivo release, precipitation, carrier-mediated and passive transport, and saturable metabolism. The program uses a mechanistic PK model to deconvolute in vivo release and establish a direct correlation with in vitro dissolution. The advantage of a mechanistic deconvolution method was illustrated using metoprolol as an example. Metoprolol is metabolized by CYP2D6 and displays nonlinear pharmacokinetics. The Loo-Riegelman and mechanistic deconvolution methods were both used to establish an IVIVC for three ER metoprolol formulations. The comprehensive mechanistic absorption model allowed a correlation to be established between in vitro and in vivo release that met the criteria for internal and external predictability, while the correlation using the Loo-Riegelman method met only the criteria for internal predictability.
Global expectations for the IVIVC were discussed by Vivian Gray in her talk entitled Need for Harmonized IVIVC Guidances to Expedite Global Drug Approvals? She described the compendial chapters and regulatory guidances that addressed IVIVC. She discussed the draft EMA Guidance "Quality of Oral Modified-Release Products," which is a comprehensive guidance on the attributes of the optimal dissolution method and describes a Level A validation for IVIVC. She reviewed the ICH treatment of IVIVC in the ICH document Q6A - Specifications: Test Procedures and Acceptance Criteria for New Drug Substances and Products, Decision Tree \# 7: Setting Acceptance Criteria for Drug Products. She could not find evidence that the guidances or chapters for IVIVC needed harmonization, as the information in these documents was not contradictory. She stressed the importance of developing discriminating dissolution methods that would yield clinically relevant specifications. She encouraged the use of Design of Experiments (DoE) to show the discriminatory power of the method for critical quality attributes.

Following active discussions concerning the guidances issued by different regulatory agencies, Dr. Patrick Marroum, formerly with the FDA Office of New Drug Quality Assessment and the Office of Clinical Pharmacology and now with Marroum Pharmaceutical Consulting, USA, discussed USA Regulatory Experience on IVIVC-New and Generic Drugs and shared with the audience case studies of FDA IVIVC reviews. He discussed the fact that most of the formulations with successful IVIVC models had been modified-release formulations (especially extendedrelease), controlled-release injectables, implants, vaginal rings, and drug-eluting stents. IVIVC failures were primarily immediate-release products and transdermal drug delivery systems. He also pointed out that most IVIVC submissions had originated from innovator companies and that generic manufacturers had not often attempted to establish an IVIVC. He emphasized the importance of having a robust dissolution method for a successful IVIVC and that the chances of success were greater when the rate-limiting step for drug absorption was release of drug from a formulation. It is also important to use several formulations with different release characteristics (at least a $10 \%$ difference). The audience learned that bioavailability studies with large populations are not a prerequisite for a successful IVIVC, since studies ranging from 6 to 36 subjects have been used for FDA submissions. However, human data are required for regulatory consideration with the only exception being DES formulations. In addition, while Level A correlations are the best route for an FDA biowaiver, he illustrated a successful Level C correlation that utilized eight lots in multiple parallel studies to validate an IVIVC. A successful IVIVC can lead to FDA waivers for bioavailability studies to bridge clinical and commercial formulations or approve manufacturing site or equipment changes, formulation changes, and source of raw material. Dissolution specifications are also easier to 
define when an IVIVC has been established. Wider specifications may be allowed by the regulatory agency based on what the correlation predicts. Further, a validated IVIVC can impart in vivo meaning to the in vitro dissolution test and thus provide a valuable tool for quality control.

\section{REFERENCES}

1. Extended Release Oral Dosage Forms: Development, Evaluation, and Application of In Vitro/In Vivo
Correlations; Guidance for Industry; U.S. Department of Health and Human Services, Food and Drug Administration, Center for Drug Evaluation and Research (CDER), U.S. Government Printing Office: Washington, DC, 1997.

2. <1088> In Vitro and In Vivo Evaluation of Dosage Forms. In The United States Pharmacopeia and National Formulary USP 36-NF 31; The United States Pharmacopeial Convention, Inc.: Rockville, MD, 2013. 